\title{
Predictive factors of response to splenectomy in children with immune thrombocytopenic purpura
}

\author{
Tamer Fakhry(1), MD; Hassan S. Badr(2), MD
}

\author{
(1) Pediatric Surgery Unit, Menoufiya University Hospitals. \\ (2) Pediatric Department, Menoufiya University Hospitals.
}

Background: Immune thrombocytopenic purpura (ITP) is an acquired disorder of unknown cause in which autoantibodies are directed against antigens on the platelet membrane. These platelets are then cleared at an accelerated rate by tissue macrophages in the spleen and other components of the reticuloendothelial system, leading to decreased platelet counts. Medical treatment for ITP includes corticosteroids, intravenous immunoglobulin (IVIG), or intravenous anti-Rho(D) immune globulin (WinRho). Splenectomy is effective in $60 \%$ to $90 \%$ of children with chronic ITP. Up to $30 \%$ of children with chronic ITP will continue to have bleeding problems after a splenectomy, and there is the small but present risk of overwhelming postsplenectomy infection, even with the proper immunizations and penicillin prophylaxis. Possible lack of response to splenectomy" and "inability to predict response to splenectomy" highlight the need for more potent predictors of response to splenectomy in children with chronic ITP.

Patients and methods: This study was conducted as a retrospective analysis of all pediatric patients 18 years of age or younger at Menoufia University Hospital with ITP who received medical treatment and eventually required splenectomy between January 2003 and June 2012. Data from files of 24 patients included initial CBC including platelets count and mean platelet volume and initial response to steroid therapy. Also we studied their sex, age, platelet count, years of thrombocytopenia and associated disease. Time of splenectomy and the response to medical therapy were also analyzed to assess their association with splenectomy response.

Results: We found that 16 cases (67\%) (11 male and 5 female) achieved complete remission while 8 cases (33\%)(3 male and 5 female) didn't, but 3 of them achieved partial remission. The mean age at splenectomy of responders and non-responders was $9.906 \pm 1.3193$ and $11.938 \pm 2.4413$ respectively, the initial platelets count at diagnosis was $22.4825 \pm 11.52027$ and $22.1813 \pm 9.23428$ respectively, there were a statistically significant difference when regarding age in years at time of splenectomy (age was higher in non-responders), and platelets count just before splenectomy (higher in non-responders), while other variables did not significantly differ.

Conclusion: We concluded that steroid response period is the main predictive factor for response to splenectomy in the study. Also initial MPV, and age in years can be considered as a predictive factor while other variables are not.

\section{Introduction:}

Immune thrombocytopenic purpura (ITP) is an acquired disorder of unknown cause in which autoantibodies are directed against antigens on the platelet membrane. These platelets are then cleared at an accelerated rate by tissue macrophages in the spleen and other components of the reticuloendothelial system, leading to decreased platelet counts. ${ }^{1}$ ITP usually occurs in children between 2 and 10 years of age with the sudden appearance of bruising and/or bleeding, and $60 \%$ of patients have a history of a recent previous infection. $^{2}$ The acute form of this disorder often occurs after a viral infection and is caused by an immune response in which 
antiplatelet antibodies are produced primarily in the spleen. ${ }^{3}$ Most children with immune thrombocytopenic purpura (ITP) experience spontaneous resolution of their disease without medical intervention. ${ }^{4}$ About $70 \%$ to $80 \%$ of children have the acute form of the disease, which, by definition, entails a full recovery of platelets $>150,000 / \mu \mathrm{L}$ within 6 months with or without therapy. In contrast, $20 \%$ to $30 \%$ of children develop chronic ITP with continued platelet counts $<150,000 / \mu \mathrm{L}$ for $>6$ months. $^{2}$ Medical therapy is initiated generally when a patient has a platelet count of $<10,000 / \mu \mathrm{L}$ with cutaneous bleeding or in the presence of a concomitant or preexisting condition that increases the risk of bleeding. ${ }^{5}$

Medical treatment for ITP includes corticosteroids, intravenous immunoglobulin (IVIG), or intravenous anti-Rho (D) immune globulin (WinRho). Other drug therapy options for children who have proven refractory to standard treatment include rituximab, cyclosporine, vincristine, vinblastine, danazol and others. Patients with persistent bleeding symptoms requiring repeated or continuous medical therapy, and those who develop chronic ITP may benefit from splenectomy. Splenectomy is effective in $60 \%$ to $90 \%$ of children with chronic ITP. Up to $30 \%$ of children with chronic ITP will continue to have bleeding problems after a splenectomy, and there is the small but present risk of overwhelming postsplenectomy infection, even with the proper immunizations and penicillin prophylaxis. ${ }^{6}$ In very young children, especially children under 2 years of age, it is wise to attempt to defer splenectomy because of the greater risk of overwhelming postsplenectomy infection. ${ }^{7}$

Possible lack of response to splenectomy" and "inability to predict response to splenectomy" highlight the need for more potent predictors of response to splenectomy in children with chronic ITP. ${ }^{8}$

We have, therefore in this study, undertaken a review of splenectomies for ITP at our institution in an attempt to identify preoperative factors that could guide pediatricians and surgeons in determining which children are most likely to have a favorable response to splenectomy.

\section{Patients and methods:}

This study was conducted as a retrospective analysis of all pediatric patients 18 years of age or younger at Menoufia University Hospital with ITP who received medical treatment and eventually required splenectomy between January 2003 and June 2012. Inpatient and outpatient records were reviewed for the patients selected, and all data were compiled in a spread sheet. Twenty four consecutive patients with chronic primary ITP refractory to ordinary treatment; prednisone, IV anti-D immunoglobulin and IV immunoglobulin (did not achieve complete remission after one year of treatment), who had been splenectomized, were included in the study.

All patients in this study had chronic ITP, defined as thrombocytopenia (platelet count $<100,000 / \mathrm{mm} 3$ ) that persisted for a minimum of 6 months without other clinical or laboratory findings that could support another etiology of the thrombocytopenia and presence of abundant megakaryocytes in bone marrow aspiration according to ASH panel. ${ }^{9}$ Exclusion criteria included positive markers for HBV, HCV and HIV; antinuclear antibodies and Coomb's test and patients that did not need treatment. Each patient in our study received at least 1 course of steroid therapy during the course of the disease and some patients were treated with intravenous IG, anti-D immunoglobulin. All of the patients eventually went on to receive open splenectomy. A retrospective study was done from their files to see initial CBC including platelets count and mean platelet volume and initial response to steroid therapy. Also we studied their sex, age, platelet count, years of thrombocytopenia, associated disease, time of splenectomy and the response to medical therapy were also analyzed to assess their association with splenectomy response.

Response to splenectomy was considered as complete (CR) if platelet count rose to $>100$ $\times 109 / 1$. Remission was defined as complete response persisting after all treatments were discontinued for one year, platelet count of $50,000 / \mu \mathrm{L}$ or more but less than $100,000 / \mu \mathrm{L}$ 
constituted a partial response (PR), and platelet count less than $50,000 / \mu \mathrm{L}$ was defined as no response (NR). ${ }^{4}$

Responses to medical therapy were categorized according to the peak platelet count with treatment. Splenectomy responses were defined by the platelet count on postoperative day 1, at initial follow-up (at least 2 weeks after splenectomy), and at the latest follow-up time-point available in the medical record.

\section{Results:}

Table (1) shows that there were a statistically significant difference when comparing the cases responding to splenectomy with other cases who did not respond to splenectomy during the follow up period regarding age in years at time of splenectomy and platelets count just before splenectomy $\times 10^{3}$, while other variables did not significantly differ.

In Table (2) when the variable has a beta coefficient $>0.5$ as steroid response period (Beta coefficient $=0.736$ : that means every 1 day increase in steroid response period there is chance about $73.6 \%$ to respond to splenectomy) ( $\mathrm{P}<0.05)$. So, it is the main predictor of the study. Also initial MPV and age in years can be considered as a predictive factors while other variables are not.

\section{Discussion:}

Splenectomy is a treatment option for children with ITP refractory to medical therapy or for those who require continuous drug therapy in order to maintain acceptable platelet levels. The first reported splenectomy for ITP was performed in Germany in 1916 and splenectomy continues to be an important treatment for patients with uncontrolled chronic ITP or with life-threatening hemorrhage complicating acute ITP. 10

This study included twenty four consecutive patients (14 male and 10 female) with chronic primary ITP refractory to ordinary treatment (medical treatment did not achieve complete remission after one year of treatment), who had been splenectomized.

We found that 16 cases (67\%) (11 male and 5 female) achieved complete remission while 8 cases (33\%) (3 male and 5 female) didn't, but 3 of them achieved partial remission. The mean age at splenectomy of responders and non-responders was 9.906 \pm 1.3193 and $11.938 \pm 2.4413$ respectively, the initial platelets count at diagnosis was $22.4825 \pm 11.52027$ and $22.1813 \pm 9.23428$ respectively.

When we compared between the cases responding to splenectomy with other cases who did not respond to splenectomy during the follow up period regarding all the parameters included in the study we found that there were a statistically significant difference when regarding age in years at time of splenectomy (age was higher in nonresponders), and platelets count just before splenectomy (higher in non-responders), while other variables did not significantly differ.

When we studied the correlation between response to splenectomy and the variables included to find out their role in predicting the response we found that steroid response period is the main predictor of the study. Also initial MPV, and age in years can be considered as predictive factors while other variables are not.

Previous studies have reported a 60$90 \%$ immediate remission rate. It has been difficult to identify predictors of response to splenectomy, and studies have reported conflicting findings. Ramenghi et al. ${ }^{11}$ found that an excellent response to either steroids or IVIG with platelet counts $>150,000 / \mu \mathrm{L}$ was predictive of a positive response to splenectomy. Coon ${ }^{12}$ showed that any response to steroid therapy $>50,000 / \mu \mathrm{L}$ was suggestive of a success with splenectomy (72\% success vs. $24 \%$ failure). Both groups found that a poor response to steroids was not predictive of a poor response to splenectomy and these findings agree with the finding of our study. In contrast, Wood et $\mathrm{al}^{13}$. found that a poor response to steroids with platelets $<50,000 / \mu \mathrm{L}$ was indicative of a CR to splenectomy with platelets $>150,000$ / $\mu \mathrm{L}$ and that a good response to steroids was not predictive of a favorable response to 
Table (1): Comparison between responders and non responders to splenectomy.

\begin{tabular}{|l|l|l|l|}
\hline & \multicolumn{1}{|c|}{$\begin{array}{c}\text { Responders } \\
\mathrm{N}=16 \text { mean } \pm \text { SD }\end{array}$} & $\begin{array}{c}\text { Non-responders } \\
\mathrm{N}=8 \text { mean } \pm \text { SD }\end{array}$ & P-value \\
\hline Initial Plat X103 & $22.4825 \pm 11.52027$ & $22.1813 \pm 9.23428$ & 0.949 \\
\hline Initial MPV fl & $9.2625 \pm 1.51925$ & $8.1825 \pm 1.06226$ & 0.087 \\
\hline Steroid response period days & $45.31 \pm 34.290$ & $29.88 \pm 29.342$ & 0.289 \\
\hline Age year & $9.906 \pm 1.3193$ & $11.938 \pm 2.4413$ & 0.014 \\
\hline time of splenectomy month & $23.56 \pm 7.848$ & $22.38 \pm 6.479$ & 0.716 \\
\hline $\begin{array}{l}\text { Platelets count just before } \\
\text { splenectomy } \mathrm{x} 10^{3}\end{array}$ & $43.4356 \pm 26.31408$ & $74.3700 \pm 17.73368$ & 0.007 \\
\hline
\end{tabular}

Table (2): Correlation between different factors and response to splenectomy.

\begin{tabular}{|l|l|c|l|}
\hline \multicolumn{1}{|c|}{ Model } & Standardized Coefficients & $\mathrm{T}$ & P-value \\
\hline Steroid response period days & 0.736 & 3.070 & 0.008 \\
\hline Initial MPV fl & 0.721 & 2.613 & 0.020 \\
\hline Age year & 0.568 & 2.831 & 0.013 \\
\hline time of splenectomy month & $0.378-$ & 2.054 & 0.058 \\
\hline Initial Plat X10 & $0.358-$ & 1.745 & 0.101 \\
\hline Sex & 0.216 & 1.226 & 0.239 \\
\hline platelets just befor splenectomy x1033 & 0.118 & 0.530 & 0.604 \\
\hline
\end{tabular}

splenectomy. Holt et al ${ }^{14}$. reviewed 24 patients treated with prednisone for ITP and found no significant association between response to prednisone and response to splenectomy. However, neither the steroid regimen nor the method of categorizing steroid responses is explicitly described in the study. Hemmila et $\mathrm{al}^{7}$. also found no correlation of steroid response to splenectomy response in 17 patients. However, $94 \%$ of patients in this series had either a "good" or "excellent" response to corticosteroids.

Some recent studies on children with ITP have reported that a good response to IVIG predicted a good response to splenectomy, but a poor response to IVIG was not predictive of a poor response to splenectomy. Few children in our study received IVIG and anti-D Ig, but the number of patients did not support meaningful statistical analysis.

Limitations of this study include the small patient number $(n=24)$, limited follow-up for some patients included in the study, and the inability to examine steroid dose and exact duration of therapy. Validation of these findings would require a prospective study.

\section{Conclusion:}

It is important to identify preoperative factors that could guide pediatricians and surgeons in determining which children are most likely to have a favorable response to splenectomy. When we studied the correlation between response to splenectomy and the variables included to find out their role in predicting the response we found that steroid response period is the main predictor of the study. Also initial MPV, and age in years can be considered as predictive factors while other variables are not.

\section{References:}

1- Kuhne T, Buchanan GR, Zimmerman S, et al: A prospective comparative study of 2540 infants and children with newly diagnosed idiopathic thrombocytopenic purpura (ITP) from the Intercontinental Childhood ITP Study Group. J Pediatr 2003; 143: 605-608.

2- Lusher JM, Iyer R: Idiopathic thrombocytopenic purpura in children. Semin Thromb Hemost 1977; 3: 175-199. 
3- Coon WW: Surgical aspects of splenic disease and lymphoma. Curr Probl Surg 1998; 35: 543-646.

4- Di Paola JA, Buchanan GR: Immune thrombocytopenia. Pediatr Clin North Am 2002; 49(5): 911-928.

5- Provan D, Stasi R, Newland AC, et al: International consensus report on the investigation and management of primary immune thrombocytopenia. Blood 2010; 115: 168-186.

6- Kurtzberg J, Stockman $3^{\text {rd }}$ JA: Idiopathic autoimmune thrombocytopenic purpura. Adv Pediatr 1994; 41: 111-134.

7- Hemmila MR, Foley DS, Castle VP, Hirschl $\mathrm{RB}$ : The response to splenectomy in pediatric patients with idiopathic thrombocytopenic purpura who fail high-dose intravenous immune globulin. J Pediatr Surg 2000; 35(6): 967-972.

8- Wood JH, Patrick DA, Hays T, Ziegler MM: Predicting response to splenectomy in children with immune thrombocytopenic purpura. J Pediatr Surg 2010; 45(1): 967-972.
9- ASH 43 ${ }^{\text {rd }}$ Annual Meeting and Exposition, 2001, Orlando.

10- Kliegman RM, Behrman RE, Jenson HB, et al: Nelson Textbook of Pediatrics Saunders, Philadelphia (Pa) (2007).

11- Ramenghi U, Amendola G, Farinasso L, Giordano P, Loffredo G, Nobili B, et al: Splenectomy in children with chronic ITP: Long-term efficacy and relation between its outcome and responses to previous treatments. Pediatr Blood Cancer 2006; 47(5 suppl): 216-223.

12- Coon WW: Splenectomy for idiopathic thrombocytopenic purpura. Surg Gynecol Obstet 1987; 164: 225-229.

13- Wood JH, Partrick DA, Hays T, Ziegler MM: Predicting response to splenectomy in children with immune thrombocytopenic purpura. J Pediatr Surg 2010; 45: 140.

14- Holt D, Brown J, Terrill K, et al: Response to intravenous immunoglobulin predicts splenectomy response in children with immune thrombocytopenic purpura. Pediatrics 2003; 111: 87-90. 